\title{
Effect of Virtual Reality-based Training Program on Patients with Mild Cognitive Impairment
}

\author{
Geun-Ho Lee ${ }^{1}$ \\ ${ }^{1}$ Professor, Department of Neurology, College of Medicine, Dankook University, Korea, \\ dneuro@hotmail.com
}

\begin{abstract}
Exercise programs using virtual reality (VR) been proven effective in relieving symptoms of dementia and delaying the deterioration of it. Nintendo Wii (Wii), a VR game, has been proposed as an easy-to-install and accessible device because it is cheaper and lighter than conventional VR systems. This study aimed to define the effect of a rehabilitation program using Wii, upon the level of cognitive function, depression, and independent performance of daily activities, in early dementia people. The research target population were 40 old men/women suffering with mild cognitive impairment (MCI), who were randomly allocated to the VR-game group and the control group, with each group consisting of 20 patients. The VR-game group was provided with a training program using Wii, while the control group received no intervention except conventional elderly care. The intervention period was 12 weeks, during which time a total of 36 sessions were provided, at the rate of three sessions per week of 15 minutes each. The cognitive function was found to have improved in the VR-game group, whereas it got worse in the control group after the program. A comparison of the scores in the Geriatric Depression Scale and the ADL(activities of daily living) showed statistically significant decrease in the VR-game group. The findings in this study suggest that the Wii-based VR training program would reduce depression and enhance or maintain the quality of life in older people with MCI, while delaying the development of moderate dementia. The result may be attributed to the fact that the participation in the program was regarded as a leisure activity by the participants with MCI. Wii can replace the expensive and large-scale existing VR system in the field of the exercise intervention treatment of MCI patients.
\end{abstract}

Keywords: Mild Cognitive Impairment, Alzheimer's Disease, Virtual Reality, Depression, Activities of Daily Living

\section{Introduction}

The South Korean society is aging so rapidly, subsequently that the prevalence of dementia is rapidly increasing as a common geriatric disease[1]. Mild cognitive impairment (MCI) is an early form of dementia, with preserved function of daily activities despite memory disorder and intact judgment and cognitive function[2]. In other words, it is an intermediate stage between dementia and normal, where the affected individual experiences reduced cognitive function compared to those in the same age group and education level, while daily living activities and social role performances maintained. Among the peoples aged over 65 years, the MCI prevalence is $10-20 \%$. Although approximately $1-2 \%$ in the normal group develops dementia, $10-15 \%$ in the MCI group advances to dementia[3]. If MCI is left untreated for a long time, cognitive function is impaired and other problematic behaviors are likely to increase the probability of developing moderate dementia that prevents independent living, as well as

Received: August 11, 2020; $1^{\text {st }}$ Review Result: September 26, 2020; $2^{\text {nd }}$ Review Result: November 14, 2020 Accepted: December 28, 2020 
facilitate the onset[4]. Thus, the goal of the intervention for MCI patients is to delay the development of dementia and to enhance the quality of life among patients. One method to enable this is that of using a combination of continuous drug treatment and regular exercise[5].

Depression was found to be one of the psychological symptoms of MCI in $20.1-44.3 \%$ of the patients[6]. On comparing populations who developed Alzheimer's dementia and normal individuals, depressive symptoms were observed $67 \%$ of cases of Alzheimer's dementia population and $31 \%$ of normal population. It was reported that the depression prevalence was higher in older people with reduced cognitive function than in older people with normal cognitive function and that the life quality was higher in the population of high cognitive function and a low level of depression[7]. It is not only the cognitive abilities and the ADL in Alzheimer's dementia patients that are degraded by the way of depression, but the family members who care for them are also adversely impacted. Older people with dementia are prone to loneliness, helplessness, depression, and isolation, owing to the lack of social communication. To motivate them in such a negative emotional state, health care services that can promote the willingness to receive treatment are necessary.

Recently, virtual reality (VR)-based interventions, where an activity can be performed in a VR setting as in reality, have been applied in rehabilitation treatment to solve psychosocial problems such as depression, deteriorated quality of life, and stress[8]. VR allows interaction with a virtual environment in a 3D space, via physical sensations, response and behavior, and cognition. The method is currently used in the rehabilitation of patients with various neurology patients[9]. Due to cognitive decline, it is difficult for dementia patients to imitate an exercise program or to perform an exercise program by remembering movements which are demonstrated to follow. It is reported that virtual reality has a positive effect on the treatment of dementia patients.

However, despite these advantages, VR systems require intervention by experts because it is difficult to attach equipment to the body and difficult to use. Furthermore, the equipment that can realize VR is also very heavy, which require a lot of cost or size, making it difficult to apply them in general social facilities. Therefore, the necessity of easy and economic VR program is emerging. Nintendo Wii (Wii), a VR game device developed by Nintendo, is a very simple structure that can be easily manipulated and economical. It is reported as an effective approach to neurological diseases such as stroke, which can promote visual and auditory biofeeding, physical activity, and hand movement, and can induce pleasant and active participation. Interventional programs using Wii can provide joy and comfort to the receivers of rehabilitation as it includes games meant for leisure, the result of which may be a positive psychological change[10].

In domestic studies using Wii, there were studies on the effects of changes in upper limb function, visual perception, balance sensory function, or lower limb muscle strength in cerebral vascular diseases, spinal cord diseases, and Parkinson's disease patients. A typical virtual reality device, Wii is much simpler and cheaper than conventional virtual reality devices, and can be easily distributed to patients, is expected to induce interest and increase the effect of exercise therapy by promoting the game results.

Nevertheless, there is a general lack of studies in which Wii-based programs are provided to older people with MCI. Besides, they were studies that were evaluated using only non-quantitative means of measurement. In this study, the effect of Wii-based training program on the cognitive abilities, depressive symptoms and ADL in MCI patients was investigated in order to suggest a VR treatment approach that can encourage their participation. The hypotheses of this study are as follows:1) Can Wiibased VR program improve the cognitive function of MCI patients? 2) Can it alleviate depression in MCI patients? 3) Can it improve the daily life activity of MCI patients? In short, will the Wii be as effective as other expensive and large VR programs?

\section{Materials and Methods}

\subsection{Subjects}


The subject recruited were 40 geriatric patients aged $\geq 65$ years, diagnosed with MCI based on the score of 17-22 by the Montreal Cognitive Assessment (MoCA) and according to the Petersen criteria, among the patients visiting the Dementia Clinic at D Hospital for reduced cognitive function during the period between March 2018 and August 2019. The MoCA is an assessment tool specially developed for the diagnosis of MCI. The required time is approximately $10 \mathrm{~min}$, and the sensitivity and specificity of MoCA for MCI diagnosis are $80-100 \%$ and $50-76 \%$, respectively, with $25 / 26$ cut-off. All subjects enrolled in this study were given a diagnosis based on a detailed patient-centered interview of medical history and reports of neurological examination and tests, blood tests, cognitive function assessment, and brain CT or MRI.

All patients passed individual demographic investigation and screening tests composed by the MiniMental State Examination (MMSE-K). The MMSE-K consisted of seven domains: orientation to time, place, registration, recall, attention, calculation, language and visuospatial questions. Scores below 23 can indicate severe ( $\leq 9$ points), moderate (10-18 points), or mild (19-23 points) cognitive impairment.

With the cut-off as MMSE-K $\leq 23$ or $19 \leq$ according to age and education level, the patients diagnosed with MCI, and those under medication for depression treatment were excluded from the study. The patients whose blood test results showed thyroid dysfunction and vitamin deficiency, severe renal disease, uncontrolled diabetes, hepatic failure that may affect the cognitive function, history of drinking three or more glasses of alcoholic drinks per day, and history of stroke were excluded from the study.

\subsection{Methods}

The patients were divided into the VR-game group and control group based on random allocation, with each group containing 20 patients. The VR-game group was provided with a training program using Nintendo Wii, while the control group received no intervention except conventional elderly care. The control group subjects were blind about which type of intervention received. All participants of game group were fully explained the game method and started the intervention after the demonstration. When the subjects had difficulty in knowing the game method, they were encouraged to continue to participate in the intervention after showing repeated demonstrations and explanations. During the intervention, a therapist conducted continuous safety management right next to him for reasons such as falls.

All subjects were assessed by neurologist doctors having than seven-year experience in Dankook university hospital. The doctors were made unaware of research goals. The intervention comprised 36 treatments in total, each session lasting $15 \mathrm{~min}$; the treatment was provided three times a week for 12 weeks. The measurement consisted of Clinical Dementia Rating (CDR), the Short Form of Geriatric Depression Scale (GDS), and ADL (Seoul-Instrumental Activities of Daily Living) before the intervention. There was a follow-up study after 12 weeks treatment. There were no changes in the number of participants.

All participants were requested to sign a informed consent. Dankook University Hospital's institutional review board (2018-08-005-007) approved this study and it follows the principles stated in the Declaration of Helsinki.

\subsection{Instruments}

\subsubsection{Clinical Dementia Rating}

The Clinical Dementia Rating (CDR) was made to evaluate the cognitive function. It was expanded in South Korea and modified to the Korean version of CDR. The goal of the CDR is to state the criteria for the level of dementia in clinical studies and to make a good assessment of the cognitive and social functions. The CDR is composed of six items. 


\subsubsection{Depression Scale}

The Short Form of GDS was developed by Sheikh \& Yesavage (1986) as an assessment tool in the form of a questionnaire evaluating depression in older people. The items of the questionnaire reflect the logical, emotional, cognitive, physical, and social aspects in older people to examine the overall state of depression. In this study, the Korean version of the GDS was used. This tool is composed of a total of 15 questions based on a dichotomous scale; "yes" is assigned 0 points and "no", 1 points; the negative questions are reversescored. The level of depression is determined with a base point of 5 ; scores $\geq 5$ indicate an increase in the depressive symptoms. The confidence coefficient, i.e., Cronbach's $\alpha$, of the tool at the time of development was 0.88 .

The BDI is a depression scale developed to be used as an assessment tool to screen depression and to measure therapeutic effects[11]. It is composed of 21 questions including those evaluating the depressive symptoms. Each question is scored on a 0-3 scale and there are four sentences describing the severity of a depressive symptom; the participant is guided to select one sentence that is closest to his/her personal experience. An increase in the total score can be interpreted as an increase in the level of depression. A score from 0-9 indicates a state without depression, 10-15 mild, 16-23 moderate, and $\geq 24$ severe depression. In this study, a score $\geq 16$ was the cut-off for the state of depression.

\subsubsection{Seoul-Instrumental Activities of Daily Living}

A tool named as The Seoul-Instrumental Activities of Daily Living (S-IADL) is applied to examine whether an older person with a weakened body and mind could independently perform the ADL and whether he or she requires care at a facility or institution[12]. The scores are based on a 0-3 scale for 15 domains, where 0 point is given for "requires no help at all", 1 point for "requires a little help", 2 points for "requires a lot of help", and 3 points for "unable to perform the activity independently". Thus, an increase in the score indicates an increase in the severity of reduced cognitive function and mental and physical abilities, which is interpreted as increased dependency.

\subsubsection{Nintendo Wii}

Nintendo Wii (Wii) is a game created by the Japanese company Nintendo. It is a device with the function of movement recognition, which can be connected to the TV and operated using a remote controller. The game involves virtual reality interaction while being simple and easy to play so that individuals of all ages can enjoy using it. To use Nintendo Wii, its main unit set and unique software are required. The main unit set comprises the main unit, a remote controller, a remote controller plus, a nunchaku, and an AC adapter exclusive for use with Wii, while the sensor bar installed above the screen computerizes the kinetic data of the user movements measured through the remote controller.

The game provides an environment similar to reality, and the user can adjust his or her movements based on the movements of the character on screen. Appropriate feedback is given to the user via the visual and auditory stimulations of the performance, and he or she can adjust the direction, range, and speed of the movements through the interactions with the character on screen. To perform the Wii game in an appropriate response to the feedback, the user should have adequate control over judgment, reasoning, and problem-solving abilities (Nintendo of Korea, Co. Ltd., 2010).

The Wii Sports Resort used as the VR program in this study was the version released for 2009-2020 by Nintendo in South Korea, which consists of a range of sports activities including water sports and common ball sports. Considering the education level and the cultural characteristics of the subjects and the fact that geriatric dementia patients find it difficult to perform challenging tasks, four sports (fencing, bowling, table tennis, and frisbee) were selected from the available 12 sports in the intervention program. The four sports were of the type that could be played with ease using a single remote controller and would stimulate the subjects' interest in the task performance through instant feedback. 


\subsection{Statistical Analysis}

The values of the experimental results were analyzed by the SPSS ver. 12.0 for Windows (SPSS Inc., Chicago, IL, USA). The statistical significance was set to .05. For the test of normality, the ShapiroWilks test was performed, and for the pretest of intergroup homogeneity after the normality has been confirmed, the two-sided $\chi^{2}$ test was performed. For comparison of the data before and after the treatment period as of depression, cognitive function, and ADL, the Wilcoxon signed-rank test was used. Differences between the groups as of the effects of intervention were analyzed by Mann-Whitney u-test.

\section{Results}

\subsection{Demographic Data of Subjects}

In the VR-game group, the sex distribution of the participants was $65 \%$ men and $35 \%$ women, mean age of $71.45 \pm 6.33$ years ( $40 \%$ aged $65-70,40 \%$ aged $71-75$, and $20 \%$ aged $76-80$ years), and in the control group, it was $60 \%$ men and $40 \%$ women, a mean age of $72.12 \pm 6.48$ years (40\% aged 65-70, $40 \%$ aged $71-75,20 \%$ aged $76-80$ years).

The general characteristics showed less significant differences between the VR-game and the control group. The homogeneity between the two groups was compared before and after participation in the program. The MMSE-K score before the start of the study was $22.25 \pm 1.42$ in the experimental group and $22.38 \pm 1.29$ in the control group. There was statistically less significant differences in age, gender, weight, disease duration, or cognitive function was shown between two groups ( $p>.05$ ), as shown in [Table 1].

\subsection{Changes in Cognitive Function according to VR-based Game Training Program}

The change in cognitive function reflected in CDR scores showed significant improvement after intervention in the VR-game group, whereas it was worse in the control group. The mean CDR was found to have improved in the VR-game group by $.39( \pm .05)$, from $.72( \pm .02)$ before program to .33 $( \pm .07)$ after the program, whereas it got worse in the control group by .06 $( \pm .03)$, from $.71( \pm .03)$ before program to $.77( \pm .04)$ after the program. Statistically significant difference was found between two groups $(\mathrm{t}=8.143, \mathrm{p}<.05)$ as shown in [Table 2] and [Table 3]. The hypothesis of "Can Wii-based virtual reality program improve the cognitive function of MCI patients?" was satisfied.

\subsection{Changes in Depression according to VR-based Game Training Program}

The Short form-GDS scores before and after participating were used comparing between the VRgame group and control group. The result showed a decline in the scores of the VR-game group from $6.21 \pm 1.64$ before intervention to $3.80 \pm 1.11$ after intervention, while the decline in scores of the control group was from $6.10 \pm 1.57$ to $5.96 \pm 1.13$. On comparing the level of decrease between the two groups, the decrease in the scores in the VR-game group was more than that in the control group by $2.27 \pm .08$, indicating a significant improvement through the program.

The BDI score, likewise, showed a significant intergroup difference $(F=24.09, p<.05)$. The mean score before treatment was $10.48 \pm 3.64$, while the follow-up monitoring after treatment showed a decrease in the score from $10.51 \pm 4.04$ to $6.64 \pm 3.19$ in the VR-game group and from $10.45 \pm 4.10$ to $9.94 \pm 4.23$ in the control. To summarize, the VR-game subjects showed a significant improvement in the depression scale after the program compared to the control group, as stated in [Table 2] and [Table 3]. The hypothesis of "Can Wii game program alleviate depression in MCI patients?" was satisfied. 


\subsection{S-IADL}

The mean S-IADL score in the VR-game group before treatment $(13.41 \pm 3.09)$ markedly decreased to $9.90 \pm 3.81$ after treatment, which shows a remarkable improvement of individual independence as shown in [Table 2]. The score in the control group increased by $.16( \pm 3.20)$, from $13.18( \pm 3.52)$ to 13.34 $( \pm 3.64)$, to indicate a significant intergroup difference $(\mathrm{p}<.05)$, as stated in both [Table 2] and [Table 3]. It means that the hypothesis of "Can Wii improve the daily life activity of MCI patients?" was satisfied.

\section{Discussions}

A few studies on the effects of virtual reality games on cognitive abilities and depression of MCI patients are present. Therefore, it was the purpose of this study investigating the effects of the virtual reality game, Nintendo Wii, on the cognitive function, depression, and ADL, in older people with MCI. As conventional VR devices have been so expensive and heavy, there existed the necessity of easy and inexpensive VR program until now. Therefore, the ultimate purpose of this study was to define whether Wii game can be effective enough to replace conventional VR therapy. The result shows that this purpose was met.

The subjects in this study were the old people with MCI, who had normal ability to perform ADL while residing in a community, suspected of cognitive impairment as per their MMSE-K score within the range of 21-23. The mean score of cognitive function was $22.30( \pm .24)$ across all subjects, so they were not diagnosed with dementia although reduced cognitive function was shown in comparison to healthy individuals. Considering many reports saying that $10-15 \%$ in the MCI group advances to dementia[3], leaving the subjects in this state could lead to a continuous fall in cognitive function and subsequent dementia, indicating the need for an intervention to strengthen the cognitive function.

The cognitive function in the subjects who underwent the VR game-based treatment resulted statistically significant increase in the CDR score after the program. This coincided with the result of studies where therapeutic Wii game was used as intervention for older people with mild dementia residing in a facility[13]. The fact that the score of cognitive function decreased in the control group that received no intervention for dementia confirmed that the cognitive function continued to decrease in the patients with mild dementia who did not receive treatment, and that activity to constantly stimulate cognitive function was necessary.

Despite the difference in subjects between the previous studies on older people diagnosed with dementia and the present study on the individuals with MCI, a stage preceding dementia, it has been shown that the application of a program to strengthen cognitive function in older people could prevent the deterioration in cognitive function, leading to a big decrease in the risk of disease progression in this high-risk dementia group.

A significant fall in the scores of GDS and BDI after the intervention was found only in the VR-game group, while the control group showed an increase in depression. The result was in line with that of the preceding study[14]. It may be explained by the fact that older people participating in the VR-gamebased intervention regarded it as leisure activity rather than as a treatment so that they enjoyed it, which led to a fall in depression. In most previous studies, the older people with reduced cognitive function due to dementia were reported to experience anxiety and depression so that an intervention program with a multilateral approach was necessary to prevent such symptoms.

In a preceding study, the application of a VR program in older people was reported to have improved their gait, which subsequently reduced depression[15]. The findings in previous studies and this study collectively indicate that participating in a VR program is regarded as an enjoyable activity by older people, so the program is effective in improving the depressive symptoms induced by MCI. 
Many studies conducted in South Korea, a VR program was recieved about three times a week for more than six weeks in dementia patients admitted in facilities or hospitals, and the results showed a significant improvement in depressive symptoms, abnormal behaviors, and cognitive function after the program[16]. However, the subjects in these studies were dementia patients admitted to a care facility or hospital, who had not been categorized based on precise clinical diagnostic criteria, so it was difficult to determine the therapeutic effects between the type and level of cognitive impairment.

On the other hand, the MCI patients in this study were primarily selected using randomized sampling, from patients diagnosed using a systematically structured set of clinical diagnostic criteria among older people aged $\geq 65$ years, residing in a specific region. In addition, by providing a standardized treatment to the subjects continuously for a set period of time, the effect of the treatment on psychological and physical activities of daily living was evaluated in a relatively objective way. The intervention was found to have a positive effect on the subjects, as the S-IADL score in the VR-game group significantly decreased more than that in the control group. This remarks would be because the activity of performing a task to gain a score by participating in the program elicited feelings of competition as well as interaction with other participants, which led to interest and positive feedback from one another to create a sense of accomplishment.

The regular VR exercise had led to an improvement in balance ability and gait velocity, encouraging social participation. The result implied that the VR program had stimulated interest in exercise as a continuous approach. The program was also effective in minimizing the boredom that may be experienced during exercise, while allowing the participants to have fun by increasing the selfmotivation in task performance[17]. For subjects who experienced stress but lost interest in a long-term rehabilitation treatment, a VR-game-based intervention helped reduce the stress in performing a task, while allowing them to feel emotionally relaxed and have fun. The studies on the effect of a training program on dementia patients have mainly focused on evaluating the improvement in the behavioral and psychological symptoms, including depression, anxiety, and abnormal behaviors[18]. Very few studies have reported improvements in the actual performance of daily activities in dementia patients so that the clinical significance of the findings in this study is high.

Physiologically, regular exercise through the game improved cardiorespiratory fitness, which increased the amount and activity of the main neurotransmitters such as serotonin, norepinephrine, and dopamine, while reducing the level of cortisol and cardiovascular response to emotional stress as well as inflammatory response[19]. Last but not least, a high level of cardiorespiratory fitness is regarded to get on the brain-derived neurotrophic factor (BDNF) and neurogenesis in the hippocampus, resulting in a good effect on emotion and ADL[20].

Since patients who are immersed in Wii games are more competitive and eager to achieve more points based on the results of the game, they can activate the reward circuitry to increase dopamine secretion by placebo effect, which can improve depression and become active in treatment. It can be said that stress and anxiety worries are relieved through games. As a reflection of this, 45 percent of the subjects who participated in the game wanted to buy a game console and continue their exercise even after the research was completed.

Wii, being a start example of using virtual reality, is much simpler and cheaper than conventional virtual reality devices, and is expected to increase the effectiveness of exercise therapy by promoting stimulation as a result of the game. The Wii game console program, which does not take up much space, is inexpensive, mobile and easily placeable at home and in facilities, and has the advantage of motivating patients with virtual reality, will require more research for future physical therapy for MCI patients. Conclusively, virtual reality treatment using Wii game consoles could be an good and safe intervention treatment method in terms of cost-effectiveness, which can alleviate depression and improve quality of life by improving MCI patients' cognitive function and increasing activity. In other words, Wii can replace the expensive and large-scale existing VR system in the field of the exercise intervention 
treatment of MCI patients under conditions of limited space or low economic conditions, such as at home or elderly center.

The limitations of this study are as follows: the relatively less sample size prevented generalizing the results, and lack of session-by-session measurements restricted the intervention period to 12 weeks. Lastly, the research on Wii as a means of physiotherapy intervention for MCI patients has only recently been reported, so it is difficult to find suitable comparison targets due to few previous studies. It is recommended that further studies recruit a larger number of participants and perform the analysis based on the measurements taken at each session. The robustness of the treatment should also be investigated through follow-up monitoring, and a study should be conducted to generalize the findings of this study.

\section{Tables}

[Table 1] General Characteristics of Subjects

\begin{tabular}{cccc}
\hline Variable & VR-game group (N=20) & Control group (N=20) & $p$ \\
\hline Gender (male:female) & $13: 7$ & $12: 8$ & .89 \\
\hline Age (year) & $71.45 \pm 6.33$ & $72.12 \pm 6.48$ & .79 \\
\hline Weight (kg) & $67.8 \pm 9.5$ & $69.2 \pm 10.7$ & .56 \\
\hline Height (cm) & $168.5 \pm 10.5$ & $165.8 \pm 8.8$ & .66 \\
\hline MMSE-K & $22.25 \pm 1.42$ & $22.38 \pm 1.29$ & .85 \\
\hline Disease duration (month) & $11.5 \pm 6.6$ & $12.1 \pm 4.5$ & .61 \\
\hline
\end{tabular}

[Table 2] The Comparison of Results Data within Each Group

\begin{tabular}{ccccccc}
\hline \multirow{2}{*}{ Variable } & \multicolumn{3}{c}{ VR-game group } & \multicolumn{3}{c}{ Control group } \\
\cline { 2 - 7 } & Pre-test & Post-test & $p$ & Pre-test & Post-test & $p$ \\
\hline CDR & $.72 \pm .02$ & $.33 \pm .07$ & $.001^{*}$ & $.71 \pm .03$ & $.77 \pm .04$ & .811 \\
\hline $\begin{array}{c}\text { Short form- } \\
\text { GDS }\end{array}$ & $6.21 \pm 1.64$ & $3.80 \pm 1.11$ & $.002^{*}$ & $6.10 \pm 1.57$ & $5.96 \pm 1.13$ & .892 \\
\hline BDI & $10.51 \pm 4.04$ & $6.64 \pm 3.19$ & $.012^{*}$ & $10.48 \pm 3.10$ & $9.94 \pm 4.23$ & .903 \\
\hline S-IADL & $13.41 \pm 3.09$ & $9.9 \pm 3.81$ & $.033^{*}$ & $13.18 \pm 3.52$ & $13.34 \pm 3.64$ & .798 \\
\hline$*$ p $<.05$ & & & & & &
\end{tabular}

${ }^{*} \mathrm{p}<.05$

CDR, Clinical Dementia Rating; BAI, Beck Anxiety Inventory; Short formGDS, geriatric depression scale; S-IADL,Seoul-Instrumental activities of daily living

[Table 3] The Comparison of Result Data between Members of Each Group

\begin{tabular}{|c|c|c|c|c|c|}
\hline \multicolumn{2}{|c|}{ Variable } & \multirow{2}{*}{$\begin{array}{c}\text { VR-game group } \\
.72 \pm .02\end{array}$} & \multirow{2}{*}{$\begin{array}{c}\text { Control group } \\
.71 \pm .03\end{array}$} & \multirow{2}{*}{$\frac{\mathrm{Z}}{-.10}$} & \multirow{2}{*}{$\begin{array}{c}p \\
.988\end{array}$} \\
\hline \multirow{2}{*}{ CDR } & Pre-test & & & & \\
\hline & Post-test & $.33 \pm .07$ & $.77 \pm .04$ & -2.55 & $.002 *$ \\
\hline Short form-GDS & Pre-test & $6.21 \pm 1.64$ & $6.10 \pm 1.57$ & -.12 & .976 \\
\hline
\end{tabular}




\begin{tabular}{cccccc}
\hline & Post-test & $3.80 \pm 1.11$ & $5.96 \pm 1.13$ & -2.52 & $.012^{*}$ \\
\hline \multirow{2}{*}{ BDI } & Pre-test & $10.51 \pm 4.04$ & $10.48 \pm 3.10$ & -.11 & .975 \\
\cline { 2 - 6 } & Post-test & $6.64 \pm 3.19$ & $9.94 \pm 4.23$ & -2.50 & $.002^{*}$ \\
\hline \multirow{2}{*}{ S-IADL } & Pre-test & $13.41 \pm 3.09$ & $13.18 \pm 3.52$ & -.10 & .966 \\
\cline { 2 - 6 } & Post-test & $9.90 \pm 3.81$ & $13.34 \pm 3.64$ & -2.44 & $.015^{*}$ \\
\hline
\end{tabular}

$* \mathrm{p}<.05$; CDR, Clinical Dementia Rating; BDI, Beck Depression Inventory; Short formGDS, geriatric depression scale; S-IADL,Seoul-Instrumental activities of daily living

\section{Conclusions}

This paper showd the study result of investigation about the effect of Wii-game-based treatment of 36 sessions provided to geriatric patients with MCI within three months. The results of the cognitive and depression questionnaires showed substantial improvement after the treatment, which coincided with the results of the previous studies. In this study, the Wii-game was shown to significantly improve the S-IADL score. The VR-based training program in this study encouraged participation of older people such that the self-motivated activities reduced their depression and enhanced the quality of life, thus delaying the development of moderate dementia. The significance of this study lies in the fact that the effect of Wii, a VR-based training program, has been proven, so the program may be suggested as an intervention method for older people with MCI. In conclusion, it can be concluded that Wii can replace the expensive and large-scale existing VR system in the field of the exercise intervention treatment of MCI patients under conditions of limited space or low economic conditions, such as at home or elderly center. Further large-scale studies of a larger patient population are needed to confirm this result.

\section{References}

[1] A. Wino, L. Jonsson, J. Bond, M. Prince, B.Winblad, Alzheimer Disease International, The worldwide economic impact of dementia 2010, Alzheimer's \& Dementia, (2013), Vol.9, No.1, pp.1-11, https://doi.org/10.1016/j.jalz.2012.11.006.

[2] R. C. Petersen, R. Doody, A. Kurz, R. C. Mohs, J. C. Morris, P. V. Rabins, K. Ritchie, M. Rossor, L. Thal, B. Winblad, Current Concepts in Mild Cognitive Impairment, Archives of Neurology, (2001), Vol.58, pp.1985-1992.

[3] W. D. Brenowitz, W. A. Kukull, S. A.A. Beresford, S.E.Monsell, E.C. Williams, Social relationships and risk of incident mild cognitive impairment in US alzheimer's disease centers, Alzheimer Disease and Associated Disorders, (2014), Vol.28, No.3, pp.253-260.

[4] M. Lee, A study on the care experience of the elderly with dementia and the quality of life of the family, Asia-pacific Journal of Convergent Research Interchange, (2018), Vol.4, No.1, pp. 53-61.

[5] T. F. Hughes, J. T. Becker, C. W. Lee, C. H. Chang, M. Ganguli, Independent and combined effects of cognitive and physical activity on incident MCI, Alzheimer's \& Dementia, (2015), Vol.11, No.11, pp.1377-1384.

[6] F. Panza, V. Frisardi, C. Capurso, A D'Introno, A. M. Colacicco, B. P. Imbimbo, A. Santamato, G. Vendemiale, D. Seripa, A. Pilotto, A.Capurso, V. Solfrizzi, Late-life depression, mild cognitive impairment and dementia: possible continuum?, The American Journal of Geriatric Psychiatry, (2010), Vol.18, No.2, pp.98-116.

[7] M. S. Kim, S. Y. Yoon, E. Y. Oh, Relationships among cognition, activities of daily living and depression in persons with decreased memory, Journal of Korean Public Health \& Nursing, (2012), Vol.26, No.3, pp.404-416.

[8] B. S. Lange, P. Requejo, S. M. Flynn, A. A. Rizzo, F. J. Valero-Cuevas, L. Baker, C. Winstein, The potential of virtual reality and gaming to assist successful aging with disability, Physical Medicine and Rehabilitation Clinics of North America, (2010), Vol.21, No.2, pp.339 -356.

[9] D. K. Lee, E. K. Kim, Y. N. Kim, Y. Kim, T. Hwang, Effects of virtual reality training program on balance and lower 
muscular strength of Parkinson's disease patients, The Journal of Korean Physical Therapy, (2013), Vol.25, No.2, pp.96-102.

[10] B. S. Lange, P. Requejo, S. M. Flynn, A. A. Rizzo, F. J. Valero-Cuevas, L. Baker, C. Winstein, The potential of virtual reality and gaming to assist successful aging with disability, Physical Medicine and Rehabilitation Clinics of North America, (2010), Vol.21, No.2, pp.339-356.

[11] J. Yoon, D. Jon, H. Hong, N. Hong, J. Seok, Reliability and validity of the Korean version of inventory for depressive symptomatology, Journal of Korean Society for Depressive and Bipolar Disorders, (2012), Vol.10, pp.131-151.

[12] Y. Yang, H.Yang, Y. Hong, J. Kim, M. Park, H. Na, I. Han, S. Kim, Activities of daily living and dementia, Dementia and Neurocognitive Disorders, (2012), Vol.11, No.2, pp.29-37.

[13] A. Leahey, J. F. Singleton, Utilizing Therapeutic recreation to empower persons with Alzheimer's in a day center, Therapeutic Recreation Journal, (2011), Vol.45, No.2, pp.135-146.

[14] J. Chesler, S. McLaren, B. Klein, S. Watson, The effects of playing Nintendo Wii on depression, sense of belonging and social support in Australian aged care residents: a protocol study of a mixed methods intervention trial, BMC Geriatrics, (2015), Vol.15, No.1, pp. 1-8.

[15] S. Lee, S. Lee, Y. Kim, J. Chae, The effects of 4 weeks training using virtual reality game on balance and gait ability, depression of elderly people, Journal of the Korean Proprioceptive Neuromuscular Facilitation Association, (2012), Vol.10, No.2, pp. 41-46.

[16] E. Lee, Who are those happy elderly people?, Asia-pacific Journal of Convergent Research Interchange, (2018), Vol.4, No.2, pp.43-50.

[17] J. F. Esculier, J. Vaudrin, P. Bériault, K. Gagnon, L. E. Tremblay, Home-based balance training programme using Wii fit with balance board for Parkinson's disease: a pilot study, Journal of Rehabilitation Medicine, (2012), Vol.44, No.2, pp.144-150.

[18] K.W. Cho, S.C. Choe, The Effect of the elderly's motivation to participate in lifelong education on their life satisfaction, Asia-pacific Journal of Convergent Research Interchange, (2019), Vol.5, No.2, pp.59-67.

[19] R. K. Dishman, X. Sui, T. S. Church, G. A. Hand, M. H. Trivedi, S. N. Blair, Decline in cardiorespiratory fitness and odds of incident depression, American Journal of Preventive Medicine, (2012), Vol.43, No.4, pp.361-368.

[20] C. M. C. Nascimento, J. R. Pereira, L. P. Andrade, M. Garuffi, C. Ayan, D. S. Kerr, L. L. Talib, M. R.Cominetti, F. Stella, Physical exercise improves peripheral BDNF levels and cognitive functions in mild cognitive impairment elderly with different bdnf Val66Met genotypes, Journal of Alzheimers Disease, (2015), Vol.43, No.1, pp.81-91. 